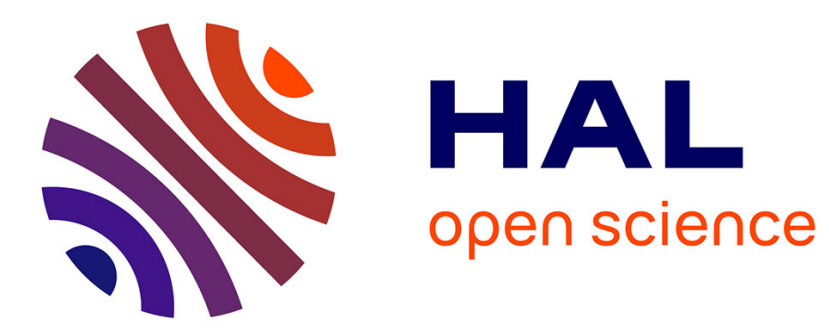

\title{
Chern character, loop spaces and derived algebraic geometry.
}

Bertrand Toen, Gabriele Vezzosi

\section{To cite this version:}

Bertrand Toen, Gabriele Vezzosi. Chern character, loop spaces and derived algebraic geometry.. Algebraic Topology, Springer Berlin Heidelberg, pp.331-354, 2009, Abel Symposia, 978-3-642-01199-3. 10.1007/978-3-642-01200-6_11. hal-00772859

\section{HAL Id: hal-00772859 \\ https://hal.science/hal-00772859}

Submitted on 11 Jan 2013

HAL is a multi-disciplinary open access archive for the deposit and dissemination of scientific research documents, whether they are published or not. The documents may come from teaching and research institutions in France or abroad, or from public or private research centers.
L'archive ouverte pluridisciplinaire HAL, est destinée au dépôt et à la diffusion de documents scientifiques de niveau recherche, publiés ou non, émanant des établissements d'enseignement et de recherche français ou étrangers, des laboratoires publics ou privés. 


\title{
Note of Chern character, loop spaces and derived algebraic geometry
}

\author{
Bertrand Toën \\ Institut de Mathématiques de Toulouse \\ UMR CNRS 5219 \\ Université Paul Sabatier, Bat. 1R2 Toulouse Cedex 9 \\ France
}

\author{
Gabriele Vezzosi \\ Dipartimento di Matematica Applicata \\ Università di Firenze \\ Italy
}

March 2008

\begin{abstract}
In this note we present a work in progress whose main purpose is to establish a categorified version of sheaf theory. We present a notion of derived categorical sheaves, which is a categorified version of the notion of complexes of sheaves of $\mathcal{O}$-modules on schemes, as well as its quasi-coherent and perfect versions. We also explain how ideas from derived algebraic geometry and higher category theory can be used in order to construct a Chern character for these categorical sheaves, which is a categorified version of the Chern character for perfect complexes with values in cyclic homology. Our construction uses in an essential way the derived loop space of a scheme $X$, which is a derived scheme whose theory of functions is closely related to cyclic homology of $X$. This work can be seen as an attempt to define algebraic analogs of elliptic objects and characteristic classes for them. The present text is an overview of a work in progress and details will appear elsewhere.
\end{abstract}

\section{Contents}

1 Motivations and objectives

2 Categorification of homological algebra and dg-categories

3 Loop spaces in derived algebraic geometry

\section{Motivations and objectives}

The purpose of this short note is to present a construction of a Chern character defined for certain sheaves of categories rather than sheaves of modules (e.g. vector bundles or coherent sheaves). This is part of a more ambitious project to develop a general theory of categorical sheaves, in the context of algebraic geometry but also in topology, which is supposed to be a categorification of the theory of 
sheaf of modules. Our original motivations for starting such a project come from elliptic cohomology, which we now explain briefly.

\section{From elliptic cohomology to categorical sheaves}

To any (complex oriented) generalized cohomology theory $E_{*}$ (defined on topological spaces) is associated an integer called its chromatic level, which by definition is the height of the corresponding formal group. The typical generalized cohomology theory of height zero is singular cohomology and is represented by the Eleinberg-MacLane spectrum $H \mathbb{Z}$. The typical generalized cohomology theory of height 1 is complex K-theory which is represented by the spectrum $B U \times \mathbb{Z}$. A typical cohomology theory of height 2 is represented by an elliptic spectrum and is called elliptic cohomology. These elliptic cohomologies can be combined altogether into a spectrum tmf of topological modular forms (we recommend the excellent survey [Lu1] on the subject). The cohomology theories $H \mathbb{Z}$ and $B U \times \mathbb{Z}$ are rather well understood, in the sense that for a finite $\mathrm{CW}$ complex $X$ is possible to describe the groups $[X, H \mathbb{Z}]$ and $[X, B U \times \mathbb{Z}]$ easily in terms of the topology of $X$. Indeed, $[X, H \mathbb{Z}] \simeq H^{0}(X, \mathbb{Z})$ is the group of continuous functions $X \longrightarrow \mathbb{Z}$. In the same way, $[X, B U \times \mathbb{Z}]=K_{0}^{\text {top }}(X)$ is the Grothendieck group of complex vector bundles on $X$. As far as we know, it is an open question to describe the group $[X, \mathrm{tmf}]=E l l_{0}(X)$, or the groups $[X, E]$ for some elliptic spectrum $E$, in similar terms, e.g. as the Grothendieck group of some kind of geometric objects over $X$ (for some recent works in this direction, see [Ba-Du-Ro] and [St-Te]).

It has been observed by several authors that the chromatic level of the cohomology theories $H \mathbb{Z}$ and $B U \times \mathbb{Z}$ coincide with a certain categorical level. More precisely, $[X, H \mathbb{Z}]$ is the set of continuous functions $X \longrightarrow \mathbb{Z}$. In this description $\mathbb{Z}$ is a discrete topological space, or equivalently a set, or equivalently a 0 -category. In the same way, classes in $[X, B U \times \mathbb{Z}]$ can be represented by finite dimensional complex vector bundles on $X$. A finite dimensional complex vector bundle on $X$ is a continuous family of finite dimensional complex vector spaces, or equivalently a continuous map $X \longrightarrow \underline{\text { Vect }}$, where $\underline{\text { Vect }}$ is the 1-category of finite dimensional complex vector spaces. Such an interpretation of vector bundles can be made rigorous if $\underline{V e c t}$ is considered as a topological stack. It is natural to expect that $[X, \mathrm{tmf}]$ is related in one way or another to 2-categories, and that classes in $[X, \mathrm{tmf}]$ should be represented by certain continuous applications $X \longrightarrow 2-\underline{V e c t}$, where now $2-\underline{V e c t}$ is a 2-category (or rather a topological 2-stack). The notation $2-\underline{V e c t}$ suggests here that $2-\underline{V e c t}$ is a categorification of $\underline{V e c t}$, which is itself a categorification of $\mathbb{Z}$ (or rather of $\mathbb{C}$ ). If we follow this idea further the typical generalized cohomology theory $E$ of chromatic level $n$ should itself be related to $n$-categories in the sense that classes in $[X, E]$ should be represented by continuous maps $X \longrightarrow n-\underline{V e c t}$, where $n-\underline{V e c t}$ is now a certain topological $n$-stack, which is supposed to be an $n$-categorification of the $(n-1)$-stack $(n-1)-\underline{V e c t}$.

This purely formal observation relating the chromatic level to a certain categorical level is in fact supported by at least two recent results. On the one hand, J. Rognes stated the so-called red shift conjecture, which from an intuitive point of view stipulates that if a commutative ring spectrum $E$ is of chromatic level $n$ then its $K$-theory spectrum $K(E)$ is of chromatic level $n+1$ (see [Au-Ro]). Some explicit computations of $K(B U \times \mathbb{Z}$ ) proves a major case of this conjecture for $n=1$ (see $[\mathrm{Ba}-\mathrm{Du}-\mathrm{Ro}])$. Moreover, $K(B U \times \mathbb{Z})$ can be seen to be the $K$-theory spectrum of the 2-category of complex 2-vector spaces (in the sense of Kapranov-Voevodsky). This clearly shows the existence of an interesting relation between elliptic cohomology and the notion of 2-vector bundles (parametrized version of the notion 2-vector spaces), even though the precise relation remains unclear at the moment. On the other hand, the fact that topological $K$-theory is obtained as the Grothendieck group of vector bundles implies the existence of equivariant $K$-theory by using equivariant vector bundles. It is important to notice here that the spectrum $B U \times \mathbb{Z}$ alone is not enough to reconstruct equivariant $K$-theory and that the fact that complex $K$-theory is obtained from a categorical construction is used 
in an essential way to define equivariant $K$-theory. Recently J. Lurie constructed not only equivariant versions but also 2-equivariant versions of elliptic cohomology (see [Lu1, §5.4]). This means that not only an action of a group can be incorporated in the definition of elliptic cohomology, but also an action of a 2-group (i.e. of a categorical group). Now, a 2-group $G$ can not act in a very interesting way on an object in a 1-category, as this action would simply be induced by an action of the group $\pi_{0}(G)$. However, a 2-group can definitely act in an interesting manner on an object in a 2-category, since automorphisms of a given object naturally form a 2-group. The existence of 2-equivariant version of elliptic cohomology therefore suggests again a close relation between elliptic cohomology and 2-categories.

\section{Towards a theory of categorical sheaves in algebraic geometry}

The conclusion of the observation above is that there should exist an interesting notion of categorical sheaves, which are sheaves of categories rather than sheaves of vector spaces, useful for a geometric description of objects underlying elliptic cohomology. In this work we have been interested in this notion independently of elliptic cohomology and in the context of algebraic geometry rather than topology. Although our final motivations is to understand better elliptic cohomology we have found the theory of categorical sheaves in algebraic geometry interesting in its own and think that it deserves a fully independent development.

To be more precise, and to fix ideas, a categorical sheaf theory is required to satisfy the following conditions.

- To any scheme $X$ there exists a 2-category $\operatorname{Cat}(X)$, of categorical sheaves on $X$. The 2-category $\operatorname{Cat}(X)$ is expected to be a symmetric monoidal 2-category. Moreover, we want $\operatorname{Cat}(X)$ to be a categorification of the category $\operatorname{Mod}(X)$ of sheaves of $\mathcal{O}_{X}$-modules on $X$, in the sense that there is a natural equivalence between $\operatorname{Mod}(X)$ and the category of endomorphisms of the unit object of $\operatorname{Cat}(X)$.

- The 2-category $\operatorname{Cat}(X)$ comes equiped with monoidal sub-2-categories $\operatorname{Cat}_{q c o h}(X), \mathrm{Cat}_{\mathrm{coh}}(X)$ and $\operatorname{Cat}_{\text {parf }}(X)$, which are categorifications of the categories $Q \operatorname{Coh}(X), \operatorname{Coh}(X)$ and $\operatorname{Vect}(X)$, of quasi-coherent sheaves, coherent sheaves, and vector bundles. The monoidal 2-category $\mathrm{Cat}_{\text {parf }}(X)$ is moreover expected to be rigid (i.e. every object is dualizable).

- For a morphism $f: X \longrightarrow Y$ of schemes, there is a 2-adjunction

$$
f^{*}: \operatorname{Cat}(Y) \longrightarrow \operatorname{Cat}(X) \quad \operatorname{Cat}(Y) \longleftarrow \operatorname{Cat}(X): f_{*} .
$$

The 2 -functors $f^{*}$ and $f_{*}$ are supposed to preserve the sub-2-categories $\operatorname{Cat}_{q c o h}(X), \operatorname{Cat}_{\text {coh }}(X)$ and $\operatorname{Cat}_{\text {parf }}(X)$, under some finiteness conditions on $f$.

- There exists a notion of short exact sequence in $\operatorname{Cat}(X)$, which can be used in order to define a Grothendieck group $K_{0}^{(2)}(X):=K_{0}\left(\operatorname{Cat}_{\text {parf }}(X)\right.$ ) (or more generally a ring spectrum $\left.K\left(\operatorname{Cat}_{\text {parf }}(X)\right)\right)$. This Grothendieck group is called the secondary K-theory of $X$ and is expected to possess the usual functorialities in $X$ (at least pull-backs and push-forwards along proper and smooth morphisms).

- There exists a Chern character

$$
K_{0}^{(2)}(X) \longrightarrow H^{(2)}(X)
$$

for some secondary cohomology group $H^{(2)}(X)$. This Chern character is expected to be functorial for pull-backs and to satisfy some version of the Grothendieck-Riemann-Roch formula for pushforwards. 
As we will see in section $\S 2$, it is not clear how to develop a theory as above, and it seems to us that a theory satisfying all the previous requirements cannot reasonably exist. One major observation here is that the situation becomes more easy to handle if the categories $\operatorname{Mod}(X), Q \operatorname{Coh}(X), \operatorname{Coh}(X)$ and $\operatorname{Vect}(X)$ are replaced by their derived analogs $D(X), D_{q c o h}(X), D_{c o h}^{b}(X)$ and $D_{\text {parf }}(X)$. Our wanted categorical sheaf theory should then rather a be derived categorical sheaf theory, and is expected to satisfy the following conditions.

- To any scheme $X$ is associated a triangulated-2-category $D g(X)$, of derived categorical sheaves on $X$. Here, by triangulated-2-category we mean a 2-category whose categories of morphisms are endowed with triangulated structure in a way that the composition functors are bi-exacts. The 2-category $D g(X)$ is expected to be a symmetric monoidal 2-category, in way which is compatible with the triangulated structure. Moreover, we want $D g(X)$ to be a categorification of the derived category $D(X)$ of sheaves of $\mathcal{O}_{X}$-modules on $X$, in the sense that there is a natural triangulated equivalence between $D(X)$ and the triangulated category of endomorphisms of the unit object of $D g(X)$.

- The 2-category $D g(X)$ comes equipped with monoidal sub-2-categories $D g_{q c o h}(X), D g_{\text {coh }}(X)$ and $D g_{\text {parf }}(X)$, which are categorifications of the derived categories $D_{q c o h}(X), D_{c o h}^{b}(X)$ and $D_{\text {parf }}(X)$, of quasi-coherent complexes, bounded coherent sheaves, and perfect complexes. The monoidal 2-category $D g_{\text {parf }}(X)$ is moreover expected to be rigid (i.e. every object is dualizable).

- For a morphism $f: X \longrightarrow Y$ of schemes, there is a 2-adjunction

$$
f^{*}: D g(Y) \longrightarrow D g(X) \quad D g(Y) \longleftarrow D g(X): f_{*} .
$$

The 2-functors $f^{*}$ and $f_{*}$ are supposed to preserve the sub-2-categories $D g_{q c o h}(X), D g_{c o h}(X)$ and $D g_{\text {parf }}(X)$, under some finiteness conditions on $f$.

- There exists a notion of short exact sequence in $D g(X)$, which can be used in order to define a Grothendieck group $K_{0}^{(2)}(X):=K_{0}\left(D g_{\text {parf }}(X)\right.$ ) (or more generally a ring spectrum $\left.K\left(D g_{\text {parf }}(X)\right)\right)$. This Grothendieck group is called the secondary $K$-theory of $X$ and is expected to possess the usual functorialities in $X$ (at least pull-backs and push-forwards along proper and smooth morphisms).

- There exists a Chern character

$$
K_{0}^{(2)}(X) \longrightarrow H^{(2)}(X)
$$

for some secondary cohomology group $H^{(2)}(X)$. This Chern character is expected to be functorial for pull-backs and to satisfy some version of the Grothendieck-Riemann-Roch formula for pushforwards.

The purpose of these notes is to give some ideas on how to define such triangulated-2-categories $D g(X)$, how to define the secondary cohomology $H^{(2)}(X)$ and how to define the Chern character. In order to do this, we will follow closely one possible interpretation of the usual Chern character for vector bundles as being a kind of function on the loop space.

\section{The Chern character and the loop space}

The Chern character we will construct for categorical sheaves is based on the following interpretation of the usual Chern character. Assume that $X$ is a smooth complex algebraic manifold (or more generally a complex algebraic stack) and that $V$ is a vector bundle on $X$. Let $\gamma: S^{1} \longrightarrow X$ be a loop 
in $X$. We do not want to specify want we mean by a loop here, and the notions of loops we will use in the sequel is a rather unconventional one (see 3.1). Whatever $\gamma$ truely is, we will think of it as a loop in $X$, at least intuitively. We consider the pull-back $\gamma^{*}(V)$, which is a vector bundle on $S^{1}$. Because of the notion of loops we use this vector bundle is in fact locally constant on $S^{1}$, and thus is completely determined by a monodromy operator $m_{\gamma}$ on the fiber $V_{\gamma(0)}$. The trace of $m_{\gamma}$ is a complex number, and as $\gamma$ varies in $L X$ the loop space of $X$ (again the notion of loop space we use is unconventional) we obtain a function $C h(V)$ on $L X$. This function can be seen to be $S^{1}$-equivariant and thus provides an element

$$
C h(V) \in \mathcal{O}(L X)^{S^{1}}
$$

Our claim is that, if the objects $S^{1}$ and $L X$ are defined correctly, then there is a natural identification

$$
\mathcal{O}(L X)^{S^{1}} \simeq H_{D R}^{e v}(X)
$$

and that $C h(V)$ is the usual Chern character with values in the algebraic de Rham cohomology of $X$. The conclusion is that $C h(V)$ can be seen as a $S^{1}$-equivariant function on $L X$.

One enlightening example is when $X$ is $B G$ the quotient stack of a finite group $G$. The our loop space $L B G$ is the quotient stack $[G / G]$, for the action of $G$ on itself by conjugation. The space of functions on $L B G$ can therefore be identified with $\mathbb{C}(G)$, the space of class function on $G$. A vector bundle $V$ on $B G$ is nothing else than a linear representation of $G$, and the function $C h(V)$ constructed above is the class function sending $g \in G$ to $\operatorname{Tr}(g: V \rightarrow V)$. Therefore, the description of the Chern character above gives back the usual morphism $R(G) \longrightarrow \mathbb{C}(G)$ sending a linear representation to its class function.

Our construction of the Chern character for a categorical sheaf follows the same ideas. The interesting feature of the above interpretation of the Chern character is that it can be generalized to any setting for which traces of endomorphisms make sense. As we already mentioned, $D g_{\text {parf }}(X)$ is expected to be a rigid monoidal 2-category, and thus any endomorphism of an object possesses a trace which is itself an object in $D_{\text {parf }}(X) \simeq \operatorname{End}(1)$. Therefore, if we start with a categorical sheaf on $X$ and do the same construction as above, we get a sheaf (rather than a function) on $L X$, or more precisely an object in $D_{\text {parf }}(L X)$. This sheaf is moreover invariant under the action of $S^{1}$ and therefore is an object in $D_{\text {parf }}^{S^{1}}(L X)$, the perfect $S^{1}$-equivariant derived category of $L X$. This sheaf as itself a Chern character which is an element in $H_{D R}^{S^{1}}(L X)$, the $S^{1}$-equivariant de Rham cohomology of $L X$. This element is by definition the Chern character of our categorical sheaf. The Chern character should then expected to be a map

$$
C h: K_{0}^{(2)}(X) \longrightarrow H_{D R}^{S^{1}}(L X) .
$$

\section{Plan of the paper}

The main purpose of this paper is to make precise all the terms of this construction. For this we will start by the definitions of the 2-categories $D g(X), D g_{q c o h}(X)$, and $D g_{\text {parf }}(X)$, but we do not try to define $D g_{c o h}(X)$ as the notion of coherence in this categorical setting seems unclear at the moment. The objects in $D g(X)$ will be certain sheaves of dg-categories on $X$ and our approach to the notion of categorical sheaves heavily relies on the homotopy theory of dg-categories recently studied in [Ta, To2]. In a second part we will recall briefly some ideas of derived algebraic geometry and of derived schemes (and stacks) as introduced in [HAG-II, Lu2]. The loop space $L X$ of a scheme $X$ will then be defined as the derived mapping stack from $S^{1}=B \mathbb{Z}$ to $X$. We will argue that the ring of $S^{1}$-invariant functions on $L X$ can be naturally identified with $H C_{0}^{-}(X)$, the negative cyclic homology of $X$. We will also briefly explain how this can be used in order to interpret the Chern character with values in cyclic homology as we have sketched above. Finally, in a last part we will present the 
construction of our Chern character for categorical sheaves. One crucial point in this construction is to define an $S^{1}$-equivariant sheaf on the loop space $L X$. The construction of the sheaf itself is easy but the fact that it is $S^{1}$-equivariant is a delicate question which we leave open in the present work (see 4.1 and 5.1). Hopefully a detailed proof of the existence of this $S^{1}$-equivariant sheaf will appear in a future work.

\section{Categorification of homological algebra and dg-categories}

In this section we present our triangulated-2-categories $D g(X)$ of derived categorical sheaves on some scheme $X$. We will start by an overview of a rather standard way to categorify the theory of modules over some base commutative ring using linear categories. As we will see the notion of 2 -vector spaces appear naturally in this setting as the dualizable objects, exactly in the same way that the dualizable modules are the projective modules of finite rank. After arguing that this notion of 2-vector space is too rigid a notion to allow for push-fowards, we will consider dg-categories instead and show that they can be used in order to categorify homological algebra in a similar way as linear categories categorify linear algebra. By analogy with the case of modules and linear categories we will consider dualizable objects as categorified versions of perfect complexes and notice that these are precisely the smooth and proper dg-categories studied in [Ko-So, To-Va]. We will finally define the 2-categories $D g(X)$, $D g_{q c o h}(X)$ and $D g_{\text {parf }}(X)$ for a general scheme $X$ by some gluing procedure.

Let $k$ be a commutative base ring. We let $\operatorname{Mod}(k)$ be the category of $k$-modules, considered as a symmetric monoidal category for the tensor product of modules. Recall that an object $M \in \operatorname{Mod}(k)$ is said to be dualizable if the natural morphism

$$
M \otimes M^{\vee} \longrightarrow \underline{\operatorname{Hom}}(M, M)
$$

is an isomorphism (here $\underline{H o m}$ denotes the $k$-module of $k$-linear morphisms, and $M^{\vee}:=\underline{H o m}(M, k)$ is the dual module). It is easy to see that $M$ is dualizable if and only if it is projective and of finite type over $k$.

A rather standard way to categorify the category $\operatorname{Mod}(k)$ is to consider $k$-linear categories and Morita morphisms. We let $C a t(k)$ be the 2-category whose objects are small $k$-linear categories. The category of morphisms between two $k$-linear categories $A$ and $B$ in $C a t(k)$ is defined to be the category of all $A \otimes_{k} B^{o p}$-modules (the composition is obtained by the usual tensor product of bimodules). The tensor product of linear categories endow $C a t(k)$ with a structure of a symmetric monoidal 2-category for which $k$, the $k$-linear category freely generated by one object, is the unit. We have $\operatorname{End}_{C a t(k)}(k) \simeq \operatorname{Mod}(k)$, showing that $\operatorname{Cat}(k)$ is a categorification of $\operatorname{Mod}(k)$. To obtain a categorification of $M o d^{p f t}(k)$, the category of projective $k$-modules of finite type, we consider the sub-2-category of $C a t(k)$ with the same objects but for which the category of morphisms from $A$ to $B$ is the full sub-category of the category of $A \otimes_{k} B^{o p}$-modules whose objects are bi-modules $M$ such that for any $a \in A$ the $B^{o p}$-module $M(a,-)$ is projective of finite type (i.e. a retract of a finit sum of representable $B^{o p}$-modules). We let $\operatorname{Cat}^{c}(k) \subset C a t(k)$ be this sub-2-category, which is again a symmetric monoidal 2-category for tensor product of linear categories. By definition we have $\operatorname{End}_{C a t^{c}(k)}(k) \simeq \operatorname{Mod}^{p f t}(k)$. However, the tensor category $\operatorname{Mod}^{p f t}(k)$ is a rigid tensor category in the sense that every object is dualizable, but not every object in $\operatorname{Cat}^{c}(k)$ is dualizable. We therefore consider $\mathrm{Cat}^{\text {sat }}(k)$ the full sub-2-category of dualizable objects in $\mathrm{Cat}^{c}(k)$. Then, $\mathrm{Cat}^{\text {sat }}(k)$ is a rigid monoidal 2-category which is a categorification of $\operatorname{Mod}^{p f t}(k)$. It can be checked that a linear category $A$ is in $C^{\text {sat }}(k)$ if and only if it is equivalent in $C a t(k)$ to an associative $k$-algebra $B$ (as usual considered as a linear category with a unique object) satisfying the following two conditions. 
1. The $k$-module $B$ is projective and of finite type over $k$.

2. For any associative $k$-algebra $A$, a $B \otimes_{k} A$-module $M$ is projective of finite type if and only if it is so as a $A$-module.

These conditions are also equivalent to the following two conditions.

1. The $k$-module $B$ is projective and of finite type over $k$.

2. The $B \otimes_{k} B^{o p}$-module $B$ is projective.

When $k$ is a field, an object in $\operatorname{Cat}^{\text {sat }}(k)$ is nothing else than a finite dimensional $k$-algebra $B$ which is universally semi-simple (i.e. such that $B \otimes_{k} k^{\prime}$ is semi-simple for any field extension $k \rightarrow k^{\prime}$ ). In general, an object in $\mathrm{Cat}^{\text {sat }}(k)$ is a flat family of universally semi-simple finite dimensional algebras over Speck. In particular, if $k$ is an algebraically closed field any object in $C_{a t}{ }^{\text {sat }}(k)$ is equivalent to $k^{n}$, or in other words is a 2-vector space of finite dimension in the sense of Kapranov-Voevodsky (see for instance [Ba-Du-Ro]). For a general commutative ring $k$, the 2 -category $\mathrm{Cat}^{\text {sat }}(k)$ is a reasonable generalization of the notion of 2-vector spaces and can be called the 2-category of 2-vector bundles on Speck.

One major problem with this notion of 2-vector bundles is the lack of push-forwards in general. For instance, let $X$ be a smooth and proper algebraic variety over some algebraically closed field $k$. We can consider $V$ ect, the trivial 2-vector bundle of rank 1 over $X$, which is the stack in categories sending a Zariski open $U \subset X$ to the linear category $V e c t(U)$ of vector bundles over $U$. The push-forward of this trivial 2-vector bundle along the structure morphism $X \longrightarrow S p e c k$ is the $k$-linear category of global sections of $\underline{V e c t}$, or in other words the $k$-linear category $V$ ect $(X)$ of vector bundles on $X$. This is an object in $C a t(k)$, but is definitely not in $C_{a t}{ }^{s a t}(k)$. The linear category $\operatorname{Vect}(X)$ is big enough to convince anyone that it can not be finite dimensional in any reasonable sense. This shows that the global sections of a 2-vector bundle on a smooth and proper variety is in general not a 2-vector bundle over the base field, and that in general it is hopeless to expect a good theory of proper push-forwards in this setting.

A major observation in this work is that considering a categorification of $D(k)$ instead of $\operatorname{Mod}(k)$, which is what we call a categorification of homological algebra, solves the problem mentioned above concerning push-forwards. Recall that a dg-category (over some base commutative ring $k$ ) is a category enriched over the category of complexes of $k$-modules (see [Ta]). For a dg-category $T$ we can define its category of $T$-dg-modules as well as as its derived category $D(T)$ by formally inverting quasiisomorphisms between dg-modules (see [To2]). For two dg-categories $T_{1}$ and $T_{2}$ we can form their tensor product $T_{1} \otimes_{k} T_{2}$, as well as their derived tensor product $T_{1} \otimes_{k}^{\mathbb{L}} T_{2}$ (see [To2]). We now define a 2-catgeory $D g(k)$ whose objects are dg-categories and whose category of morphisms from $T_{1}$ to $T_{2}$ is $D\left(T_{1} \otimes_{k}^{\mathbb{L}} T_{2}^{o p}\right)$. The composition of morphisms is defined using the derived tensor product

$$
-\otimes_{T_{2}}^{\mathbb{L}}-: D\left(T_{1} \otimes_{k}^{\mathbb{L}} T_{2}^{o p}\right) \times D\left(T_{2} \otimes_{k}^{\mathbb{L}} T_{3}^{o p}\right) \longrightarrow D\left(T_{1} \otimes_{k}^{\mathbb{L}} T_{3}^{o p}\right) .
$$

Finally, the derived tensor product of dg-categories endows $D g(k)$ with a structure of a symmetric monoidal 2-category.

The symmetric monoidal 2-category $D g(k)$ is a categorification of the derived category $D(k)$ as we have by definition

$$
\underline{\operatorname{End}}_{D g(k)}(1) \simeq D(k) .
$$

To obtain a categorification of $D_{\text {parf }}(k)$, the perfect derived category, we consider the sub-2-category $D g^{c}(k)$ having the same of objects as $D g(k)$ itself but for which the category of morphisms from $T_{1}$ to $T_{2}$ in $D g^{c}(k)$ is the full sub-category of $D\left(T_{1} \otimes_{k}^{\mathbb{L}} T_{2}^{o p}\right)$ of bi-dg-modules $F$ such that for all $t \in T_{1}$ the 
object $F(t,-) \in D\left(T^{o p}\right)$ is compact (in the sense of triangulated categories, see [Ne]). The symmetric monoidal structure on $D g(k)$ restricts to a symmetric monoidal structure on $D g^{c}(k)$, and we have

$$
\underline{\operatorname{End}}_{D g^{c}(k)}(1) \simeq D_{\text {parf }}(k),
$$

as an object of $D(k)$ is compact if and only if it is a perfect complex. Finally, the symmetric monoidal 2-category $D g^{c}(k)$ is not rigid and we thus consider $D g^{\text {sat }}(k)$, the full sub-2-category consisting of rigid objects in $D g^{c}(k)$. By construction, $D g^{s a t}(k)$ is a rigid symmetric monoidal 2-category and we have

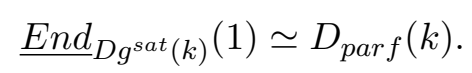

The 2-category $D g^{\text {sat }}(k)$ will be our categorification of $D_{\text {parf }}(k)$ and its objects should be thought as perfect derived categorical sheaves on the scheme Speck.

It is possible to show that an object $T$ of $D g^{c}(k)$ belongs to $D g^{s a t}(k)$ if and only if it is equivalent to an associative dg-algebra $B$, considered as usual as a dg-category with a unique object, satisfying the following two conditions.

1. The underlying complex of $k$-modules of $B$ is perfect.

2. The object $B \in D\left(B \otimes_{k}^{\mathbb{L}} B^{o p}\right)$ is compact.

In other words, a dg-category $T$ belongs to $D g^{\text {sat }}(k)$ if and only it is Morita equivalent to a smooth (condition (2) above) and proper (condition (1) above) dg-algebra $B$. Such dg-categories are also often called saturated (see [Ko-So, To-Va]).

As $D g^{s a t}(k)$ is a rigid symmetric monoidal 2-category we can define, for any object $T$ a trace morphism

$$
\operatorname{Tr}: \underline{\operatorname{End}}_{D g^{\text {sat }}(k)}(T) \longrightarrow \underline{\operatorname{End}}_{D g^{\text {sat }}(k)}(1) \simeq D_{\text {parf }}(k) .
$$

Now, the category $\underline{E n d}_{D g^{s a t}(k)}(T)$ can be naturally identified with $D\left(T \otimes_{k}^{\mathbb{L}} T^{o p}\right)_{c}$, the full sub-category of $D\left(T \otimes_{k}^{\mathbb{L}} T^{o p}\right)$ of compact objects (here we use that $T$ is saturated and the results of [To-Va, $\left.\S 2.2\right]$ ). The trace morphism is then a functor

$$
\operatorname{Tr}: D\left(T \otimes_{k}^{\mathbb{L}} T^{o p}\right)_{c} \longrightarrow D_{\text {parf }}(k)
$$

which can be seen to be isomorphic to the functor sending a bi-dg-module $M$ to its Hochschild complex $H H(T, M) \in D_{\text {parf }}(k)$. In particular, the rank of an object $T \in D g^{s a t}(k)$, which by definition is the trace of its identity, is its Hochschild complex $H H(T) \in D_{\text {parf }}(k)$.

To finish this section we present the global versions of the 2-categories $D g(k)$ and $D g^{\text {sat }}(k)$ over some base scheme $X$. We let $\operatorname{Zar} A f f(X)$ be the small site of affine Zariski open sub-schemes of $X$. We start to define a category $d g-\operatorname{cat}(X)$ consisting of the following data

1. For any $\operatorname{Spec} A=U \subset X$ in $\operatorname{Zar} A f f(X)$, a dg-category $T_{U}$ over $A$.

2. For any $\operatorname{Spec} B=V \subset \operatorname{Spec} A=U \subset X$ morphism in $Z \operatorname{ar} A f f(X)$ a morphism of dg-categories over $A$

$$
r_{U, V}: T_{U} \longrightarrow T_{V}
$$

These data should moreover satisfy the equation $r_{V, W} \circ r_{U, V}=r_{U, W}$ for any inclusion of affine opens $W \subset V \subset U \subset X$. The morphisms in $d g-\operatorname{cat}(X)$ are defined in an obvious way as families of dg-functors commuting with the $r_{U, V}$ 's.

For $T \in d g-\operatorname{cat}(X)$ we define a category $\operatorname{Mod}(T)$ of $T$-dg-modules in the following way. Its objects consist of the following data 
1. For any $\operatorname{Spec} A=U \subset X$ in $\operatorname{Zar} A f f(X)$, a $T_{U}$-dg-module $M_{U}$.

2. For any $\operatorname{Spec} B=V \subset \operatorname{Spec} A=U \subset X$ morphism in $\operatorname{Zar} A f f(X)$ a morphism of $T_{U}$-dgmodules

$$
m_{U, V}: M_{U} \longrightarrow r_{U, V}^{*}\left(M_{V}\right) .
$$

These data should moreover satisfy the usual cocyle equation for $r_{U, V}^{*}\left(m_{V, W}\right) \circ m_{U, V}=m_{U, W}$. Morphisms in $\operatorname{Mod}(T)$ are simply defined as families of morphisms of dg-modules commuting with the $m_{U, V}$ 's. Such a morphism $f: M \longrightarrow M^{\prime}$ in $\operatorname{Mod}(T)$ is a quasi-isomorphism if it is a stalkwise quasiisomorphism (note that $M$ and $M^{\prime}$ are complexes of presheaves of $\mathcal{O}_{X}$-modules). We denote by $D(T)$ the category obtained from $\operatorname{Mod}(T)$ by formally inverting these quasi-isomorphisms.

We now define a 2-category $D g(X)$ whose objects are the objects of $d g-\operatorname{cat}(X)$, and whose category of morphisms from $T_{1}$ to $T_{2}$ is $D\left(T_{1} \otimes_{\mathcal{O}_{X}}^{\mathbb{L}} T_{2}^{o p}\right)$ (we pass on the technical point of defining this derived tensor product over $\mathcal{O}_{X}$, one possibility being to endow $d g-\operatorname{cat}(X)$ with a model category structure and to use a cofibrant replacement). The compositions of morphisms in $D g(X)$ is given by the usual derived tensor product. The derived tensor product endows $D g(X)$ with a structure of a symmetric monoidal 2-category and we have by construction

$$
\underline{\operatorname{End}}_{D g(X)}(1) \simeq D(X)
$$

where $D(X)$ is the (unbounded) derived category of all $\mathcal{O}_{X}$-modules on $X$.

Definition 2.1 1. An object $T \in D g(X)$ is quasi-coherent if for any inclusion of affine open subschemes

$$
\operatorname{Spec} B=V \subset \operatorname{Spec} A=U \subset X
$$

the induced morphism

$$
T_{U} \otimes_{A} B \longrightarrow T_{V}
$$

is a Morita equivalence of dg-categories.

2. A morphism $T_{1} \longrightarrow T_{2}$, corresponding to an object $M \in D\left(T_{1} \otimes_{\mathcal{O}_{X}}^{\mathbb{I}} T_{2}^{o p}\right)$, is called quasi-coherent if its underlying complex of $\mathcal{O}_{X}$-modules is with quasi-coherent cohomology sheaves.

The sub-2-category of $D g(X)$ consisting of quasi-coherent objects and quasi-coherent morphisms is denoted by $D g_{q c o h}(X)$. It is called the 2-category of quasi-coherent derived categorical sheaves on $X$.

Let $T_{1}$ and $T_{2}$ be two objects in $D g_{q c o h}(X)$. We consider the full sub-category of $D\left(T_{1} \otimes_{\mathcal{O}_{X}}^{\mathbb{L}} T_{2}^{o p}\right)$ consisting of objects $M$ such that for any Zariski open Spec $A=U \subset X$ and any object $x \in\left(T_{1}\right)_{U}$, the induced dg-module $M(a,-) \in D\left(\left(T_{2}^{o p}\right)_{U}\right)$ is compact. This defines a sub-2-category of $D g_{q c o h}(X)$, denoted by $D g_{q c o h}^{c}(X)$ and will be called the sub-2-category of compact morphisms. The symmetric monoidal structure on $D g(X)$ restricts to a symmetric monoidal structure on $D g_{q c o h}(X)$ and on $D g_{q c o h}^{c}(X)$.

Definition 2.2 The 2-category of perfect derived categorical sheaves is the full sub-2-catgeory of $D g_{q c o h}^{c}(X)$ consisting of dualizable objects. It is denoted by $D g_{p a r f}(X)$.

By construction, $D g_{\text {parf }}(X)$ is a symmetric monoidal 2-category with

$$
\underline{\operatorname{End}}_{D g_{\text {parf }}(X)}(1) \simeq D_{\text {parf }}(X) .
$$


It is possible to show that an object $T \in D_{\text {qcoh }}(X)$ belongs to $D g_{\text {parf }}(X)$ if and only if for any affine Zariski open subscheme Spec $A=U \subset X$, the dg-category $T_{U}$ is saturated (i.e. belongs to $\left.D g^{\text {sat }}(A)\right)$.

For a morphism of schemes $f: X \longrightarrow Y$ it is possible to define a 2-adjunction

$$
f^{*}: D g(Y) \longrightarrow D g(X) \quad D g(Y) \longleftarrow D g(X): f_{*} .
$$

Moreover, $f^{*}$ preserves quasi-coherent objects, quasi-coherent morphisms, as well as the sub-2-categories of compact morphisms and perfect objects. When the morphism $f$ is quasi-compact and quasiseparated, we think that it is possible to prove that $f_{*}$ preserves quasi-coherent objects and quasicoherent morphisms as well as the sub-2-category of compact morphisms. We also guess that $f_{*}$ will preserve perfect objects when $f$ is smooth and proper, but this would require a precise investigation. As a typical example, the direct image of the unit $1 \in D g_{\text {parf }}(X)$ by $f$ is the presheaf of dg-categories sending $\operatorname{Spec} A=U \subset Y$ to the dg-category $L_{\text {parf }}\left(X \times_{Y} U\right)$ of perfect complexes over the scheme $f^{-1}(U) \simeq X \times_{Y} U$. When $f$ is smooth and proper it is known that the dg-category $L_{\text {parf }}\left(X \times_{Y} U\right)$ is in fact saturated (see $[\mathrm{To} 2, \S 8.3]$ ). This shows that $f_{*}(1)$ is a perfect derived sheaf on $Y$ and provides an evidence that $f_{*}$ preserves perfect object. These functoriality statements will be considered in more details in a future work.

\section{$3 \quad$ Loop spaces in derived algebraic geometry}

In this section we present a version of the loop space of a scheme (or more generally of an algebraic stack) based on derived algebraic geometry. For us the circle $S^{1}$ is defined to be the quotient stack $B \mathbb{Z}$, where $\mathbb{Z}$ is considered as a constant sheaf of groups. For any scheme $X$, the mapping stack $\operatorname{Map}\left(S^{1}, X\right)$ is then equivalent to $X$, as the coarse moduli space of $S^{1}$ is simply a point. In other words, with this definition of the circle there are no interesting loops on a scheme $X$. However, we will explain in the sequel that there exists an interesting derived mapping stack $\mathbb{R M a p}\left(S^{1}, X\right)$, which is now a derived scheme and which is non-trivial. This derived mapping stack will be our loop space. In this section we recall briefly the notions from derived algebraic geometry needed in order to define the object $\mathbb{R} \operatorname{Map}\left(S^{1}, X\right)$. We will also explain the relation between the cohomology of $\mathbb{R} \operatorname{Map}\left(S^{1}, X\right)$ and cyclic homology of the scheme $X$.

Let $k$ be a base commutative ring and denote by $\mathbf{S c h}_{k}$, resp. $\mathbf{S t} \mathbf{t}_{k}$, the category of schemes over $k$ and the model category of stacks over $k$ ([HAG-II, 2.1.1] or [To1, §2, 3]), for the étale topology. We recall that the homotopy category $\mathrm{Ho}\left(\mathbf{S t}_{k}\right)$ contains as full sub-categories the category of sheaves of sets on $\mathbf{S c h}_{k}$ as well as the 1-truncation of the 2-category of stacks in groupoids (in the sense of [La-Mo]). In particular, the homotopy category of stacks $\mathrm{Ho}\left(\mathbf{S t}_{k}\right)$ contains the category of schemes and of Artin stacks as full sub-categories. In what follows we will always consider these two categories as embedded in $\mathrm{Ho}\left(\mathbf{S t}_{k}\right)$. Finally, recall that the category $\mathrm{Ho}\left(\mathbf{S t}_{k}\right)$ possesses internal Hom's, that will be denoted by Map.

As explained in [HAG-II, Ch. 2.2] (see also [To1, §4] for an overview), there is also a model category $\mathbf{d S t}_{k}$ of derived stacks over $k$ for the strong étale topology. The derived affine objects are simplicial $k$-algebras, and the model category of simplicial $k$-algebras will be denoted by $\mathbf{s a l g}_{k}$. The opposite (model) category is denoted by $\mathbf{d} \mathbf{A f f}{ }_{k}$. Derived stacks can be identified with objects in the homotopy category $\mathrm{Ho}\left(\mathbf{d} \mathbf{S} \mathbf{t}_{k}\right)$ which in turn can be identified with the full subcategory of the homotopy category of simplicial presheaves on $\mathbf{d A f f}{ }_{k}$ whose objects are weak equivalences' preserving simplicial presheaves $F$ having strong étale descent i.e. such that, for any étale homotopy hypercover $U_{\bullet} \rightarrow X$ in dAff $_{k}$ ([HAG-I, Def. 3.2.3, 4.4.1]), the canonical map

$$
F(X) \longrightarrow \operatorname{holim} F\left(U_{\bullet}\right)
$$


is a weak equivalence of simplicial sets. The derived Yoneda functor induces a fully faithful functor on homotopy categories

$$
\mathbb{R S p e c}: \operatorname{Ho}\left(\mathbf{d A f f}_{k}\right) \hookrightarrow \operatorname{Ho}\left(\mathbf{d S t}_{k}\right): A \mapsto\left(\mathbb{R S p e c}(A): B \mapsto \operatorname{Map}_{\mathbf{s a l g}_{k}}(A, B)\right),
$$

where $\operatorname{Map}_{\mathbf{s a l g}_{k}}$ denotes the mapping spaces of the model category $\mathbf{s a l g}_{k}\left(\right.$ therefore $\operatorname{Map}_{\mathbf{s a l g}_{k}}(A, B) \simeq$ $\underline{\operatorname{Hom}}(Q(A), B)$, where $\underline{H o m}$ denotes the natural simplicial Hom's of $\mathbf{s a l g}_{k}$ and $Q(A)$ is a cofibrant model for $A$ ). Those derived stacks belonging to the essential image of $\mathbb{R S p e c}$ will be called affine derived stacks.

The category $\mathrm{Ho}\left(\mathbf{d S t}_{k}\right)$ of derived stacks has a lot of important properties. First of all, being the homotopy category of a model category, it has derived colimits and limits (denoted as hocolim and holim). In particular, given any pair of maps $F \rightarrow S$ and $G \rightarrow S$ between derived stacks, there is a derived fiber product stack $\operatorname{holim}(F \rightarrow S \leftarrow G) \equiv F \times{ }_{S}^{h} G$. As our base ring $k$ is not assumed to be a field, the direct product in the model category $\mathbf{d S t}_{k}$ is not exact and should also be derived. The derived direct product of two derived stacks $F$ and $G$ will be denoted by $F \times{ }^{h} G$. This derived product is the categorical product in the homotopy category $\mathrm{Ho}\left(\mathbf{d} \mathbf{S} \mathbf{t}_{k}\right)$. The category $\mathrm{Ho}\left(\mathbf{d S t}_{k}\right)$ also admits internal Hom's, i.e. for any pair of derived stacks $F$ and $G$ there is a derived mapping stack denoted as

$$
\mathbb{R M a p}(F, G)
$$

with the property that

$$
[F, \mathbb{R M a p}(G, H)] \simeq\left[F \times{ }^{h} G, H\right]
$$

functorially in $F, G$ and $H$.

The inclusion functor $j$ of commutative $k$-algebras into $\mathbf{s a l g}_{k}$ (as constant simplicial algebras) induces a pair $\left(i, t_{0}\right)$ of (left,right) adjoint functors

$$
t_{0}:=j^{*}: \operatorname{Ho}\left(\mathbf{d S t}_{k}\right) \rightarrow \operatorname{Ho}\left(\mathbf{S t}_{k}\right) \quad i:=\mathbb{L} j_{!}: \operatorname{Ho}\left(\mathbf{S t}_{k}\right) \rightarrow \operatorname{Ho}\left(\mathbf{d} \mathbf{S t} t_{k}\right) .
$$

It can be proved that $i$ is fully faithful. In particular we can, and will, view any stack as a derived stack (we will most of the time omit to mention the functor $i$ and consider $\operatorname{Ho}\left(\mathbf{S t}_{k}\right)$ as embedded in $\left.\operatorname{Ho}\left(\mathbf{d S t}_{k}\right)\right)$. The truncation functor $t_{0}$ acts on affine derived stacks as $t_{0}(\mathbb{R} \operatorname{Spec}(A))=\operatorname{Spec}\left(\pi_{0} A\right)$. It is important to note that the inclusion functor $i$ does not preserve derived internal hom's nor derived fibered products. This is a crucial point in derived algebraic geometry: derived tangent spaces and derived fiber products of usual schemes or stacks are really derived objects. The derived tangent space of an Artin stack viewed as a derived stack via $i$ is the dual of its cotangent complex while the derived fiber product of, say, two affine schemes viewed as two derived stacks is given by the derived tensor product of the corresponding commutative algebras

$$
i(\operatorname{Spec} S) \times_{i(\operatorname{Spec} R)}^{h} i(\operatorname{Spec} T) \simeq \mathbb{R} \operatorname{Spec}\left(S \otimes_{R}^{\mathbb{L}} T\right) .
$$

Both for stacks and derived stacks there is a notion of being geometric ([HAG-II, 1.3, 2.2.3]), depending, among other things, on the choice of a notion of smooth morphism between the affine pieces. For morphisms of commutative $k$-algebras this is the usual notion of smooth morphism, while in the derived case, a morphism $A \rightarrow B$ of simplicial $k$-algebras is said to be strongly smooth if the induced map $\pi_{0} A \rightarrow \pi_{0} B$ is a smooth morphism of commutative rings, and $\pi_{*} A \otimes_{\pi_{0} A} \pi_{0} B \simeq \pi_{*} B$. The notion of geometric stack is strictly related to the notion of Artin stack ([HAG-II, Prop. 2.1.2.1]). Any geometric derived stack has a cotangent complex ([HAG-II, Cor. 2.2.3.3]). Moreover, both functors $t_{0}$ and $i$ preserve geometricity. 
Let $\mathrm{B} \mathbb{Z}$ be the classifying stack of the constant group scheme $\mathbb{Z}$. We view BZ as an object of $\mathbf{S t}_{k}$, i.e. as the stack associated to the constant simplicial presheaf

$$
\mathrm{B} \mathbb{Z}: \operatorname{alg}_{k} \rightarrow \text { SSets }, R \mapsto \mathrm{B} \mathbb{Z},
$$

where by abuse of notations, we have also denoted as $\mathrm{B} \mathbb{Z}$ the classifying simplicial set, i.e. the nerve of the (discrete) group $\mathbb{Z}$. Such a nerve is naturally a pointed simplicial set, and we call 0 that point.

Definition 3.1 Let $X$ be a derived stack over $k$. The derived loop stack of $X$ is the derived stack

$$
\mathrm{L} X:=\mathbb{R} \operatorname{Map}(\mathrm{B} \mathbb{Z}, X)
$$

We will be mostly interested in the case where $X$ is a scheme or an algebraic (underived) stack. Taking into account the homotopy equivalence

$$
\mathrm{B} \mathbb{Z} \simeq S^{1} \simeq * \coprod_{* \amalg *}^{h} *,
$$

we see that we have

$$
\mathrm{L} X \simeq X \times_{X \times{ }^{h} X}^{h} X
$$

where $X$ maps to $X \times{ }^{h} X$ diagonally and the homotopy fiber product is taken in $\mathbf{d S t}_{k}$. Evaluation at $0 \in \mathrm{B} \mathbb{Z}$ yields a canonical map of derived stacks

$$
p: \mathrm{L} X \longrightarrow X .
$$

On the other hand, since the limit maps canonically to the homotopy limit, we get a canonical morphism of derived stacks $X \rightarrow \mathrm{L} X$, a section of $p$, describing $X$ as the "constant loops" in $\mathrm{L} X$.

If $X$ is an affine scheme over $k, X=\operatorname{Spec} A$ with $A$ a commutative $k$-algebra, we get that

$$
\mathrm{L} X \simeq \mathbb{R} \operatorname{Spec}\left(A \otimes_{A \otimes^{\mathbb{L}} A}^{\mathbb{L}} A\right),
$$

where the derived tensor product is taken in the model category $\mathbf{s a l g}_{k}$. One way to rephrase this is by saying that "functions" on LSpec $A$ are Hochschild homology classes of $A$ with values in $A$ itself. Precisely, we have

$$
\mathcal{O}(\mathrm{L} X):=\mathbb{R} \underline{\operatorname{Hom}}\left(\mathrm{L} X, \mathbb{A}^{1}\right) \simeq \operatorname{HH}(A, A),
$$

where $\operatorname{HH}(A, A)$ is the simplicial set obtained from the complex of Hochschild homology of $A$ by the Dold-Kan correspondence, and $\mathbb{R} \underline{H o m}$ denotes the natural enrichment of $\operatorname{Ho}\left(\mathbf{d S t}_{k}\right)$ into $\mathrm{Ho}(\operatorname{SSet})$. When $X$ is a general scheme then $\mathcal{O}(\mathrm{L} X)$ can be identified with the Hochschild homology complex of $X$, and we have

$$
\pi_{i}(\mathcal{O}(\mathrm{L} X)) \simeq H H_{i}(X) .
$$

In particular, when $X$ is a smooth and $k$ is of characteristic zero, the Hochschild-Kostant-Rosenberg theorem implies that

$$
\pi_{0}(\mathcal{O}(\mathrm{L} X)) \simeq \oplus_{i} H^{i}\left(X, \Omega_{X / k}^{i}\right) .
$$

The stack $S^{1}=\mathrm{B} \mathbb{Z}$ is a group stack, and it acts naturally on $\mathrm{L} X$ for any derived stack $X$ by "rotating the loops". More precisely, there is a model category $\mathbf{d S t}_{/ k}^{S^{1}}$, or $S^{1}$-equivariant stacks, and $\mathrm{L} X$ is naturally an object in the homotopy category $\operatorname{Ho}\left(\mathbf{d S t}_{/ k}^{S^{1}}\right)$. This way, the simplicial algebra of functions $\mathcal{O}(\mathrm{L} X)$ is naturally an $S^{1}$-equivariant simplicial algebra, and thus can also be considered as an $S^{1}$-equivariant complex or in other words as an object in $D^{S^{1}}(k)$, the $S^{1}$-equivariant derived category of $k$. The category $D^{S^{1}}(k)$ is also naturally equivalent to $D(k[\epsilon])$, the derived category of 
the dg-algebra $k[\epsilon]$ freely generated by an element $\epsilon$ of degree -1 and with $\epsilon^{2}=0$. The derived category $D^{S^{1}}(k)$ is thus naturally equivalent to the derived category of mixed complexes (see [Lo]) (multiplication by $\epsilon$ providing the second differential). When $X=\operatorname{Spec} A$ is an affine scheme, $\mathcal{O}(\mathrm{L} X)$ can be identified, as a mixed complex, with the Hoschschild complex $\operatorname{HH}(A)$, with its canonical mixed complex structure. As a consequence, we have

$$
\pi_{i}\left(\mathcal{O}(\mathrm{L} X)^{h S^{1}}\right) \simeq H C_{i}^{-}(A),
$$

where $H C^{-}$denotes negative cyclic homology and $K^{h S^{1}}$ denotes the simplicial set of homotopy fixed points of an $S^{1}$-equivariant simplicial set $K$. In other words, there is a natural identification between $S^{1}$-invariant functions on $\mathrm{L} X$ and negative cyclic homology of $X$. This statement of course can be generalized to the case of a scheme $X$.

Proposition 3.2 For a scheme $X$ we have

$$
\pi_{0}\left(\mathcal{O}(\mathrm{L} X)^{h S^{1}}\right) \simeq H C_{0}^{-}(X),
$$

where the right hand side denotes negative cyclic homology of the scheme $X$.

When $k$ is of characteristic zero and $X$ is smooth over $k$ then proposition 3.2 states that

$$
\pi_{0}\left(\mathcal{O}(\mathrm{L} X)^{h S^{1}}\right) \simeq H_{D R}^{e v}(X / k) .
$$

The even part of de Rham cohomology of $X$ can be identified with $S^{1}$-equivariant functions on the derived loop space $\mathrm{L} X$. This fact can also be generalized to the case where $X$ is a smooth DeligneMumford stack over $k$ (again assumed to be of characteristic zero), but the right hand side should rather be replaced by the (even part of) de Rham orbifold cohomology of $X$, which is the de Rham cohomology of the inertia stack $I X \simeq t_{0}(\mathrm{~L} X)$.

To finish this part, we would like to mention that the construction of the Chern character for vector bundles we suggested in section $\S 1$ can now be made precise, and through the identification of proposition 3.2 this Chern character coincides with the usual one. We start with a vector bundle $V$ on $X$ and we consider its pull-back $p^{*}(V)$ on $\mathrm{L} X$, which is a vector bundle on the derived scheme $\mathrm{L} X$. This vector bundle $p^{*}(V)$ comes naturally equipped with an automorphism $u$. This follows by considering the evaluation morphism $\pi: S^{1} \times \mathrm{L} X \longrightarrow X$, and the vector bundle $\pi^{*}(V)$. As $S^{1}=B \mathbb{Z}$, a vector bundle on $S^{1} \times \mathrm{L} X$ consists precisely of a vector bundle on $\mathrm{L} X$ together with an action of $\mathbb{Z}$, or in other words together with an automorphism. We can then consider the trace of $u$, which is an element in $\pi_{0}(\mathcal{O}(\mathrm{L} X)) \simeq H H_{0}(X)$. A difficult issue here is to argue that this function $\operatorname{Tr}(u)$ has a natural refinement to an $S^{1}$-invariant function $\operatorname{Tr}(u) \in \pi_{0}\left(\mathcal{O}(\mathrm{L} X)^{h S^{1}}\right) \simeq H C_{0}^{-}(X)$, which is the Chern character of $V$. The $S^{1}$-invariance of $\operatorname{Tr}(u)$ will be studied in a future work, and we refer to our last section below, for some comments about how this would follow from the general theory of rigid tensor $\infty$-categories.

\section{Construction of the Chern character}

We are now ready to sketch the construction of our Chern character for a derived categorical sheaf. This construction simply follows the lines we have just sketched for vector bundles. We will meet the same difficult issue of the existence of an $S^{1}$-invariant refinement of the trace, and we will leave this question as an conjecture. However, in the next section we will explain how this would follow from a very general fact about rigid monoidal $\infty$-categories. 
Let $T \in D g_{\text {parf }}(X)$ be a perfect derived categorical sheaf on some scheme $X$ (or more generally on some algberaic stack $X$ ). We consider the natural morphism $p: \mathrm{L} X \longrightarrow X$ and we consider $p^{*}(T)$, which is a perfect derived categorical sheaf on $\mathrm{L} X$. We have not defined the notions of categorical sheaves on derived schemes or derived stacks but this is rather straightforward. As in the case of vector bundles explained in the last section, the object $p^{*}(T)$ comes naturally equipped with an autoequivalence $u$. This again follows from the fact that a derived categorical sheaf on $S^{1} \times \mathrm{L} X$ is the same thing as a derived categorical sheaf on $\mathrm{L} X$ together with an autoequivalence. We consider the trace of $u$ in order to get a perfect complex on the derived loop space

$$
\operatorname{Tr}(u) \in \underline{\operatorname{End}}_{D g_{\text {parf }}(\mathrm{L} X)}(1)=D_{\text {parf }}(\mathrm{L} X) .
$$

The main technical difficulty here is to show that $\operatorname{Tr}(u)$ possesses a natural lift as an $S^{1}$-equivariant complex on $\mathrm{L} X$. We leave this as a conjecture.

Conjecture 4.1 The complex $\operatorname{Tr}(u)$ has a natural lift

$$
\operatorname{Tr}^{S^{1}}(u) \in D_{\text {parf }}^{S^{1}}(\mathrm{~L} X)
$$

where $D_{\text {parf }}^{S^{1}}(\mathrm{~L} X)$ is the $S^{1}$-equivariant perfect derived category of $\mathrm{L} X$.

The above conjecture is not very precise as the claim is not that a lift simply exists, but rather than there exists a natural one. One of the difficulty in the conjecture above is that it seems difficult to characterize the required lift by some specific properties. We will see however that the conjecture can be reduced to a general conjecture about rigid monoidal $\infty$-categories.

Assuming conjecture 4.1, we have $\operatorname{Tr}^{S^{1}}(u)$ and we now consider its class in the Grothendieck group of the triangulated category $D_{\text {parf }}^{S^{1}}(\mathrm{~L} X)$. This is our definition of the categorical Chern character of $T$.

Definition 4.2 The categorical Chern character of $T$ is

$$
C h^{c a t}(T):=\left[\operatorname{Tr}^{S^{1}}(u)\right] \in K_{0}^{S^{1}}(\mathrm{~L} X):=K_{0}\left(D_{\text {parf }}^{S^{1}}(\mathrm{~L} X)\right) .
$$

The categorical Chern character $C h^{c a t}(T)$ can be itself refined into a cohomological Chern character by using now the $S^{1}$-equivariant Chern character map for $S^{1}$-equvariant perfect complexes on $\mathrm{L} X$. We skip some technical details here but the final result is an element

$$
C h^{c o h}(T):=C h^{S^{1}}\left(C h^{c a t}(T)\right) \in \pi_{0}\left(\mathcal{O}\left(\mathrm{L}^{(2)} X\right)^{h\left(S^{1} \times S^{1}\right)}\right),
$$

where $\mathrm{L}^{(2)} X:=\mathbb{R} \operatorname{Map}\left(S^{1} \times S^{1}, X\right)$ is now the derived double loop space of $X$. The space $\pi_{0}\left(\mathcal{O}\left(\mathrm{L}^{(2)} X\right)^{h\left(S^{1} \times S^{1}\right)}\right)$ can reasonably be called the secondary negative cyclic homology of $X$ and should be thought (and actually is) the $S^{1}$-equivariant negative cyclic homology of $\mathrm{L} X$. We therefore have

$$
C h^{c o h}(T) \in H C_{0}^{-, S^{1}}(\mathrm{~L} X) .
$$

Definition 4.3 The cohomological Chern character of $T$ is

$$
C h^{c o h}(T):=C h^{S^{1}}\left(C h^{c a t}(T)\right) \in H C_{0}^{-, S^{1}}(\mathrm{~L} X):=\pi_{0}\left(\mathcal{O}\left(\mathrm{L}^{(2)} X\right)^{h S^{1} \times S^{1}}\right)
$$

defined above.

Obviously, it is furthermore expected that the constructions $T \mapsto C h^{c a t}(T)$ and $T \mapsto C h^{c o h}(T)$ satisfy standard properties such as additivity, multiplicativity and functoriality with respect to pullbacks. The most general version of our Chern character map should be a morphism of commutative ring spectra

$$
C h^{c a t}: K g(X) \longrightarrow K^{S^{1}}(\mathrm{~L} X),
$$

where $K g(X)$ is a ring spectrum constructed using a certain Waldhausen category of perfect derived categorical sheaves on $X$ and $K^{S^{1}}(\mathrm{~L} X)$ is the $K$-theory spectrum of $S^{1}$-equivariant perfect complexes on $\mathrm{L} X$. This aspect of the Chern character will again be investigated in more details in a future work. 


\section{$5 \quad$ Final comments}

\section{On $S^{1}$-equivariant trace maps}

Our conjecture 4.1 can be clarified using the language of higher categories. Recall that a $(1, \infty)$ category is an $\infty$-category in which all $n$-morphisms are invertible (up to higher morphisms) as soon as $n>1$. There exist several well behaved models for the theory of $(1, \infty)$-categories, such as simplicially enriched categories, quasi-categories, Segal categories and Rezk's spaces. We refer to [Ber1] for an overview of these various notions. What we will say below can be done in any of these theories, but, to fix ideas, we will work with $\mathbb{S}$-categories (i.e. simplicially enriched categories).

We will be using $H o(\mathbb{S}-C a t)$ the homotopy category of $\mathbb{S}$-categories, which is the category obtained from the category of $\mathbb{S}$-categories and $\mathbb{S}$-functors by inverting the (Dwyer-Kan) equivalences (a mixture between weak equivalences of simplicial sets and categorical equivalences). An important property of $\mathrm{Ho}(\mathbb{S}-\mathrm{Cat})$ is that it is cartesian closed (see [To2] for the corresponding statement for dg-categories whose proof is similar). In particular, for two $\mathbb{S}$-categories $T$ and $T^{\prime}$ we can construct an $\mathbb{S}$-category $\mathbb{R} \underline{\operatorname{Hom}}\left(T, T^{\prime}\right)$ with the property that

$$
\left[T^{\prime \prime}, \mathbb{R} \underline{\operatorname{Hom}}\left(T, T^{\prime}\right)\right] \simeq\left[T^{\prime \prime} \times T, T^{\prime}\right],
$$

where $[-,-]$ denote the Hom sets of $H o(\mathbb{S}-C a t)$. Any $\mathbb{S}$-category $T$ gives rise to a genuine category $[T]$ with the same objects and whose sets of morphisms are the connected components of the simplicial sets of morphisms of $T$.

We let $\Gamma$ be the category of pointed finite sets and pointed maps. The finite set $\{0, \ldots, n\}$ pointed at 0 will be denoted by $n^{+}$. Now, a symmetric monoidal $\mathbb{S}$-category $T$ is a functor

$$
T: \Gamma \longrightarrow \mathbb{S}-C a t
$$

such that for any $n \geq 0$ the so-called Segal morphism

$$
T\left(n^{+}\right) \longrightarrow T\left(1^{+}\right)^{n}
$$

induced by the various projections $n^{+} \rightarrow 1^{+}$sending $i \in\{1, \ldots, n\}$ to 1 and everything else to 0 , is an equivalence of $\mathbb{S}$-categories. The full sub-category of the homotopy category of functors $H o\left(\mathbb{S}-C a t^{\Gamma}\right)$ consisting of symmetric monoidal $\mathbb{S}$-categories will be denoted by $\mathrm{Ho}\left(\mathbb{S}-C a t^{\otimes}\right)$. As the category $H o(\mathbb{S}-C a t)$ is a model for the homotopy category of $(1, \infty)$-categories, the category $H o\left(\mathbb{S}-C a t^{\otimes}\right)$ is a model for the homotopy category of symmetric monoidal $(1, \infty)$-categories. For $T \in H o\left(\mathbb{S}-C a t^{\otimes}\right)$ we will again use $T$ to denote its underlying $\mathbb{S}$-category $T\left(1^{+}\right)$. The $\mathbb{S}$-category $T\left(1^{+}\right)$has a natural structure of a commutative monoid in $H o(\mathbb{S}-C a t)$. This monoid structure will be denoted by $\otimes$.

We say that a symmetric monoidal $\mathbb{S}$-category $T$ is rigid if for any object $x \in T$ there is an objetc $x^{\vee} \in T$ and a morphism $1 \rightarrow x \otimes x^{\vee}$ such that for any pair of objects $y, z \in T$, the induced morphism of simplicial sets

$$
T(y \otimes x, z) \longrightarrow T\left(y \otimes x \otimes x^{\vee}, z \otimes x^{\vee}\right) \longrightarrow T\left(y, z \otimes x^{\vee}\right)
$$

is an equivalence. In particular, the identity of $x^{\vee}$ provides a trace morphism $x \otimes x^{\vee} \rightarrow 1\left(y=x^{\vee}\right.$, $z=1$ ). Therefore, for any rigid symmetric monoidal $\mathbb{S}$-category $T$ and an object $x \in T$ we can define a trace morphism

$$
\operatorname{Tr}_{x}: T(x, x) \simeq T\left(1, x \otimes x^{\vee}\right) \longrightarrow T(1,1) .
$$

Let $T$ be a fixed rigid symmetric monoidal $\mathbb{S}$-category and $S^{1}=B \mathbb{Z}$ be the groupoid with a unique object with $\mathbb{Z}$ as automorphism group. The category $S^{1}$ is an abelian group object in categories and therefore can be considered as a group object in $\mathbb{S}$-categories. The $\mathbb{S}$-category of functors $\mathbb{R} \underline{\operatorname{Hom}}\left(S^{1}, T\right)$ is denoted by $T\left(S^{1}\right)$, and is equiped with a natural action of $S^{1}$. We consider the sub- $\mathbb{S}$-category of 
invertible (up to homotopy) morphisms in $T\left(S^{1}\right)$ whose classifying space is an $S^{1}$-equivariant simplicial set. We denote this simplicial set by $L T$ (" $L$ " stands for "loops"). It is possible to put all the trace morphisms $\operatorname{Tr}_{x}$ defined above into a morphism of simplicial sets (well defined in $H o(S S e t)$ )

$$
\operatorname{Tr}: L T \longrightarrow T(1,1) \text {. }
$$

Note that the connected components of $L T$ are in one to one correspondence with the set of equivalences classes of pairs $(x, u)$, consisting of an object $x$ in $T$ and an autoequivalence $u$ of $x$. The morphism $\operatorname{Tr}$ is such that $\operatorname{Tr}(x, u)=\operatorname{Tr}_{x}(u) \in \pi_{0}(T(1,1))$. We are in fact convinced that the trace map $\operatorname{Tr}$ can be made equivariant for the action of $S^{1}$ on $L T$, functorially in $T$. To make a precise conjecture, we consider $\mathbb{S}-\mathrm{Cat}^{\text {rig }}$ the category of all rigid symmetric monoidal $\mathbb{S}$-categories (note that $\mathbb{S}-C_{a t}{ }^{\text {rig }}$ is not a homotopy category, it is simply a full sub-category of $\mathbb{S}-C a t^{\Gamma}$ ). We have two functors

$$
\mathbb{S}-\mathrm{Cat}^{\text {rig }} \longrightarrow S^{1}-\text { SSet }
$$

to the category of $S^{1}$-equivariant simplicial sets. The first one sends $T$ to $L T$ together with its natural action of $S^{1}$. The second one sends $T$ to $T(1,1)$ with the trivial $S^{1}$-action. These two functors are considered as objects in $\mathrm{Ho}\left(\mathrm{Fun}\left(\mathbb{S}-\mathrm{Cat}^{\text {rig }}, S^{1}-S S e t\right)\right)$, the homotopy category of functors. Let us denote these two objects by $L: T \mapsto L T$ and $E: T \mapsto T(1,1)$.

Conjecture 5.1 There exists a morphism in $\operatorname{Ho}\left(\mathrm{Fun}\left(\mathbb{S}-\mathrm{Cat}^{\text {rig }}, S^{1}-\mathrm{SSet}\right)\right)$

$$
\operatorname{Tr}: L \longrightarrow E,
$$

in such a way that for any rigid symmetric monoidal $\mathbb{S}$-category $T$ the induced morphism of simplicial sets

$$
\operatorname{Tr}: L T \longrightarrow T(1,1)
$$

is the trace map described above.

It can be shown that conjecture 5.1 implies conjecture 4.1. In fact the tensor 2-categories $D g_{\text {parf }}(X)$ are the 2-truncation of natural rigid symmetric monoidal $(2, \infty)$-categories, which can also be considered as $(1, \infty)$-categories by only considering invertible higher morphisms. An application of the above conjecture to these rigid symmetric monoidal $(1, \infty)$-categories give a solution to conjecture 4.1 , but this will be explained in more detailed in a future work. To finish this part on rigid $(1, \infty)$ categories let us mention that a recent work of J. Lurie and M. Hopkins on universal properties of $(1, \infty)$-categories of 1 -bordisms seem to solve conjecture 5.1 ([Lu3]). We think to have another solution to the part of conjecture 5.1 concerned with the rigid symmetric monoidal $(1, \infty)$-categories of saturated dg-categories, which is surely enough to also imply conjecture 4.1. This again will be explained in a future work.

\section{Relations with variations of Hodge structures}

The derived loop space $\mathrm{L} X$ and the $S^{1}$-equivariant derived category $D_{\text {parf }}^{S^{1}}(\mathrm{~L} X)$ have already been studied in $[\mathrm{Be}-\mathrm{Na}]$. In this work the category $D_{\text {parf }}^{S^{1}}(\mathrm{~L} X)$ is identified with a certain derived category of modules over the Rees algebra of differential operators on $X$ (when, say, $X$ is smooth over $k$ of characteristic zero). We do not claim to fully understand this identification but it seems clear that objects in $D_{\text {parf }}^{S^{1}}(\mathrm{~L} X)$ could be identified with some kind of fltered complexes of $D$-modules on $X$. Using this identification our categorical Chern character $C h^{c a t}(T)$ probably encodes the data of the negative cyclic complex $H C^{-}(T)$ of $T$ over $X$ together with its Gauss-Manin connection and Hodge filtration. In other words, $C h^{\text {cat }}(T)$ seems to be nothing more than the variation of Hodge structures induced by the family of dg-categories $T$ over $X$. As far as we know the construction of such a structure 
of variations of Hodge structures on the family of complexes of cyclic homology associated to a family of sturated dg-categories is, up to conjecture 4.1, a new result (see however [Ge] for the construction of a Gauss-Manin connection on cyclic homology). We also think it is a remarkably nice fact that variations of Hodge structures appear naturally out of the construction of our Chern character for categorical sheaves.

It is certainly possible to describe the cohomological Chern character of 4.3 using this point of view of Hodge structure. Indeed, $H C_{0}^{-, S^{1}}(\mathrm{~L} X)$ is close to be the $S^{1}$-equivariant de Rham cohomology of $\mathrm{L} X$, and using a localization formula it is probably possible to relate $H C_{0}^{-, S^{1}}(\mathrm{~L} X)$ with $H P_{0}(X)[[t]]\left[t^{-1}\right]$, where $H P_{0}(X)$ is periodic cyclic homology of $X$ and $t$ is a formal paramater. We expect at least a morphism

$$
H C_{0}^{-, S^{1}}(\mathrm{~L} X) \longrightarrow H P_{0}(X)[[t]]\left[t^{-1}\right] .
$$

The image of $C h^{c o h}(T)$ by this map should then be closely related to the Hodge polynomial of $T$, that is $\sum_{p} C h\left(G r^{p} H C^{-}(T)\right) t^{p}$, where $G r^{p} H C^{-}(T)$ is the $p$-th graded piece of the Hodge filtration of Hochschild homology and $C h$ is the usual Chern character for sheaves on $X$.

\section{Back to elliptic cohomology?}

In the introduction of this work we mentioned that our motivation to start thinking about categorical sheaf theory was elliptic cohomology. However, our choice to work in the context of algebraic geometry drove us rather far from elliptic cohomology and it is at the moment unclear whether our work on the Chern character can really bring any new insight on elliptic cohomology. About this we would like to make the following remark. Since what we have been considering are categorified version of algebraic vector bundles, it seems rather clear that what we have done so far might have some relations with what could be called algebraic elliptic cohomology (by analogy with the distinction between algebraic and topological $K$-theory). However, the work of M. Walker shows that algebraic $K$-theory determines completely topological $K$-theory (see [Wa]), and that it is possible to recover topological $K$-theory from algebraic $K$-theory by an explicit construction. Such a striking fact suggest the possibility that an enough well understood algebraic version of elliptic cohomology could also provide some new insights on usual elliptic cohomology. We leave this vague idea for future works.

\section{References}

[Au-Ro] C. Ausoni, J. Rognes, The chromatic red-shift in algebraic K-theory, to appear in L'Enseignement Mathématique

[Ba-Du-Ro] Two-vector bundles and forms of elliptic cohomology, in Topology, Geometry and Quantum Field Theory, London Math. Soc. Lecture Note Ser. 308 Cambridge University Press (2004) 18-45.

[Be-Na] D. Ben-Zvi, D. Nadler, Loops spaces and Langlands parameters, preprint arXiv:0706.0322.

[Ber1] J. Bergner, A survey of $(1, \infty)$-categories, to appear in the proceedings of the IMA workshop on n-categories, arXiv preprint math.AT/0610239.

[Ge] E. Getzler, Cartan homotopy formulas and the Gauss-Manin connectioion in cyclic homology, in Quantum deformations of algebras and their representations, Isreal Math. Conf. Proc. 7, Bar-Ilan Univ., Ramat-Gan, 1993, 65-78.

[Ko-So] M. Kontsevich, Y. Soibelmann, Notes on A-infinity algebras, A-infinity categories and noncommutative geometry. I, arXiv Preprint math.RA/0606241. 
[La-Mo] G. Laumon and L. Moret-Bailly, Champs algébriques, A Series of Modern Surveys in Mathematics vol. 39, Springer-Verlag 2000.

[Lo] J-L. Loday, Cyclic homology, Springer, Berlin, 1992.

[Lu1] J. Lurie, A survey of elliptic cohomolgy, preprint available at http://wwwmath.mit.edu/ lurie/papers/survey.pdf

[Lu2] J. Lurie, Derived algebraic geometry, thesis.

[Lu3] J. Lurie, talk at the Abel symposium, Oslo, August 2007.

[Ne] A. Neeman, Triangulated categories, Annals of Mathematics Studies, 148. Princeton University Press, Princeton, NJ, 2001. viii+449 pp.

[St-Te] S. Stolz and P. Teichner, What is an elliptic object? In Topology, Geometry and Quantum Field Theory. Proceedings of the 2002 Oxford Symposium in Honour of the 60th Birthday of G. Segal (U. Tillmann, editor), p. 247-344, London Math. Society Lecture Note Series 308 (2004).

[Ta] G. Tabuada, Une structure de catégorie de modèles de Quillen sur la catégorie des dg-catégories, Comptes Rendus de l'Acadmie de Sciences de Paris 340 (2005), 15-19.

[To1] B. Toën, Higher and derived stacks: a global overview, to appear in Proceedings of the Seattle conference on Algebraic Geometry, arXiv preprint math.AG/0604504.

[To2] B. Toën, The homotopy theory of dg-categories and derived Morita theory, Invent. Math. 167 (2007), no. 3, 615-667.

[To-Va] B. Toën, M. Vaquié, Moduli of objects in dg-categories, to appear in Ann. Scient. Ecole Norm. Sup., arXiv preprint math.AG/0503269.

[HAG-I] B. Toën, G. Vezzosi, Homotopical algebraic geometry I: Topos theory, Advances in Math. 193 (2005), 257-372.

[HAG-II] B. Toën, G. Vezzosi, Homotopical algebraic geometry II: derived stacks and applications, to appear in Mem. AMS, arXiv preprint math.AG/0404373.

[Wa] M. Walker, Semi-topological K-homology and Thomason's Theorem, K-theory 26 (2002), 207286. 